\title{
Integrated Circuits: Symmetric Usage in Reversing Paralysis
}

\author{
Lam Kai Shun*
}

B.Sc., M.Sc. (Eng.), M.Sc. (I.T. Edu)

DOI: $10.36347 /$ sipms.2020.v07i12.002

| Received: 14.11.2020 | Accepted: 28.11.2020 | Published: 07.12.2020

*Corresponding author: Lam Kai Shun

Abstract

Original Research Article

An integrated circuit consists sets of electronic circuits on a small, flat piece of semiconductor (silicon) material. Indeed, there is usually a large numbers of tiny metal-oxide-semiconductor (MOS) transistors integrated into a small chip. The results give us those circuits in are smaller, faster and less expensive than other electronic components. There are plenty of applications for an integrated circuit (IC) in a small chip. It can function as an amplifier, oscillator, timer, microprocessor, and computer memory. This paper will discuss the development of the integrated circuit industry among the "Four Asian Dragons" (Hong Kong, Taiwan, South Korea, and Singapore) in the 1980s. In particular, this paper will review a Taiwan semiconductor company's stages of development as well as its problems. It should be noted that personal comments about this business will also be included. Importantly, with regard to reversing paralysis, the application of this author's net-seizing theory will be discussed. This constitutes to the so-called "Symmetric Reversing Paralysis Approach" for disabled and handicapped people.

Keywords: electronic circuits, silicon material, metal-oxide-semiconductor (MOS), integrated circuit (IC).

Copyright $\odot 2020$ The Author(s): This is an open-access article distributed under the terms of the Creative Commons Attribution 4.0 International License (CC BY-NC 4.0) which permits unrestricted use, distribution, and reproduction in any medium for non-commercial use provided the original author and source are credited.

\section{INTRODUCTION}

As mentioned in the abstract section, integrated circuits have become more and more important in our human history since its invention. In the following section, this author will focus on the business development of a Taiwan semi-conductor company; specifically, I.C.'s application with this author's net-seizing theory in the field of reversed paralysis, using a symmetric reversed paralysis approach.

\section{LITERATURE REVIEW \\ Developing strategic alignment}

There are numerous opinions concerning strategic alignment, especially with regard to balancing certain conflicts between information technology (IT) and business strategy. Hence, an organisation can gain a competitive advantage by properly adopting strategic alignment. That said, such measures are often a big challenge for those IT companies. For instance, a perfect alignment model may be criticised if it only focuses on policy formulation rather than execution. In fact, scholars consider strategic alignment as a continuous process. To maintain a business approach, it may be necessary to develop and re-construct the IT of organisations. Research shows that there are endless variations of IT management due to various factors.
How can organisations better develop IT in order to deploy resources and achieve strategic alignment?

\section{Viewing the alignment process from a dynamic capability perspective}

The analysis of strategic IT alignment can be viewed from different perspectives. First, alignment requires scheduling and dialogue, which is known as "intended alignment". Second, ongoing adaptation and organisational change is considered as a "strategic alignment process". The final process is "implemented alignment", which can be defined as activities linking an organisation's processes and IT infrastructure.

IT is an essential resource when it comes to maintaining an organisation's daily operations. The term "resources" has a broad definition, especially when adopting a resource-based view. The concept of dynamic capabilities lengthens the previous view, with strength and competency of resource reconfiguration being the core. Processes, positions, and paths are the dimensions of dynamic capabilities.

Processes can be defined as integration, learning, and reconfiguration within a firm. Positions and roles are the human assets of an organisation. They can be both tangible and intangible. On the other hand, 
one may categorise paths as path dependences (historical) and technological opportunities one.

In general, co-evolutionary approaches in biology can be treated as dynamic capabilities. This implies that co-evolutionary IT processes are based on their relationship within a market. Hence, in order to support other core resources, specific IT assets may need to be reconfigured according to their dynamic capability.

\section{Methodology and data collection}

A qualitative and interpretive research strategy has been selected for this study. Additionally, the research was employed using a single longitudinal case study-SEMI Corp, which is a company that engages in advanced integrated circuit packaging technology. With reference to a prior study, one can apply the lifecycle stages and methods when performing classification and identification. With this information, the study will code SEMI Corp's business tasks, centralisation, formalisation, structure, and organisational size. These include: (1) the start-up stage (1997-98), (2) the expansion stage (1999-2000), (3) the consolidation stage (2001-02), and (4) the diversification stage (2003-04).

\section{The strategic alignment process in the SEMI Corp}

First, during the start-up phase (1997-98) of SEMI Corp, each department operated in isolation for various applications. During this stage, an IT specialist was employed for computer and network maintenance. The IT specialist had to recognise manufacturing processes and enhance a small MES system to suit specific internal requirements. The employee may have also been required to possess certain business and technical skills. However, the management team thought it was only useful for the development of integrated applications (when there is a requirement). Second, for the expansion phase (1999-2000), an improper ERP system was used during the development of a database infrastructure for communication networks. Adding to this issue, an unproductive and untrustworthy MES system was developed. As a result, more IT staff were employed to implement a better solution. That said, inappropriate applications were resisted by users, and as such, the planned system implementation was delayed. In short, the in-house MES system was replaced by an outsourced MES project. As a solution, SEMI Corp planned to implement shared databases and platforms. Although IT projects were supported by larger budgets, using proper packaging systems were still underestimated by executives. Third, for the consolidation phase (200102 ), the IT team grew to 13 in order to manage the operations and maintenance of applications. The first outsourced MES system was established during this period; however, it could not adapt to certain manufacturing processes. Thus, according to different situations, both MES and ERP systems were submitted.
These events led to further acquiring knowledge about manufacturing processes and their applications. Next, the difficulty and complexity of the aforementioned systems were upgraded from the outdated product strategy alignments. Importantly, the IT manager was subsequently promoted and could attend executive meetings, and the complex material tracking system was fully supported. Finally, the diversification phase was implemented (2003-04). Overall, after merging with some other companies, 30 IT staff were employed across three different sites. Simultaneously, such mergers influenced a number of complex and rigid applications, and the MES system was rebuilt and outsourced to a larger one in size. Furthermore, the new IT director replaced existing applications with international large package software. Finally, merging with other companies had a large impact on SEMI Corp's IT infrastructure, which was underestimated by executives. Therefore, a lack emergency measures meant that necessary actions could not take place.

\section{DISCUSSION}

This essay uses dynamic capability to examine the relationship between IT alignment and business strategy. The example of SEMI Corp demonstrates how IT reconfiguration and resource development can evolve under the influence of alignment processes.

One factor that limits the development of IT strengths during business growth is the lack of associated alignment measures. For a fast-growing company, there are difficulties preserving such high levels of alignment.

The remarkable organisational changes and different IT practices is the result of constant growth strategies. Notably, the unsynchronised alignment was caused by overlooking the roles of SEMI Corp's IT department. In essence, changing a company's business strategy may affect the suitability of its IT plans. Since the IT manager was not allowed to follow different business strategies or participate in strategic planning meetings, the implemented alignment became outdated. Although SEMI Corp's executives invested in IT infrastructure, there may have been a lack of clear IT strategy, in addition to increased costs. Thus, timing is significant during the expansion stage. Therefore, it should be noted that it is difficult to develop appropriate IT or use existing systems to fit changes in business strategy.

In the consolidation stage, diverse business processes are adapted to existing applications by applying resource reconfiguration routines. Merging with other companies increases the significance of linking resource acquisition routines. Practically, IT managers must use emergent IT strategies for corresponding reactions under such circumstances [1]. The dynamic capabilities view is an excellent tool for analysing alignment processes, as it reduces disparities 
that occur during implementation phases and intended alignments. Moreover, one may relate the company's developmental trajectory with its IT resources to those dynamic capabilities. This study found that for existing IT resources, there were correspondence path dependence effects according to their IT strategies. As such, a company's earlier existing IT resources and investments can limit future actions and decisions. If there are insufficient resources, it could deteriorate certain path dependences. Moreover, there was also a lack of long-term views. The effects of insufficient resources are amplified by path dependence and nearsighted IT strategies, which originated from SEMI Corp's legacy infrastructure. Dynamic capabilities decrease path dependence constraints. Outsourced IT services can temporarily decrease path dependence, which as a result enhanced resource reconfiguration by market forces. It is possible to eliminate path dependence from IT resources via dynamic capabilities. In fact, a limited scope of learning is the major source of path dependence.

\section{Overview}

This paper expands upon the idea of strategic IT alignment as implemented and intended alignment. The trajectory of which can be linked with the dynamic capabilities of IT resource acquisition, reconfiguration, and integration. Ultimately, a clearer understanding of business vision and a long-term view of IT strategy, centred in IT resources, will be good for the overall development of IT. In fact, IT inefficiency is caused by short-term solutions and path dependence which restrict the adoption of IT and wastes investment in redundant IT resources. For companies with dynamic environments, IT managers and executives have high levels of intended alignment, though implementation is not easily recognized. It is important to develop dynamic capabilities that can be continuously adapted. Further research should look into resource-oriented IT strategies and agile application infrastructures. It would also be useful to examine the conditions that influence path dependence.

\section{DISCUSSION}

\section{Research Benefits}

By analysing SEMI Corp's use of strategic alignment to make profit gains, new IT companies might acquire valuable insight into how to improve business. In other words, they could learn from an example and overcome previously identified barriers, such as a lack of alignment (outdated business strategy combined with new IT) and path dependence. They could also attempt to solve various growth problems by narrowing the gap between intended and implemented alignment. Furthermore, IT resources could be fully implemented alongside new business strategies. Hence, spending would be wiser and more effective. Finally, in regards to dynamic capabilities, new ventures could be developed and run to a higher standard in today's extremely competitive and changing business environment.

\section{Drawbacks}

Of course, there are drawbacks to having such an alignment. First, in order to develop dynamic capabilities, a firm must overcome three obstacles: the gap created by engineering, the gap during operation, and the gap in cognition. Second, there is not much discussion on how new capabilities will be created by dynamic capabilities. Third, the research looks at the specific characteristics of firm growth in terms of dynamic capabilities. As such, it is difficult for the firm growth models to accurately portray capability development from a general point of view. Last, the discussion only focuses on internal impacts, external aspects have not been considered. As such, it is important that more attention is paid to the relation between external and internal factors. Capability can then be determined through the interaction of this relationship with the firms' operations.

\section{Limitations on the research method}

One improvement that could be made is that the research should contain more cases. For example, cases from other countries or industries. This would provide further insights and comparisons. A wider viewpoint would therefore greatly enhance the research.

\section{Suggestions for future research}

First, the research should pay more attention to how dynamic capabilities actually create new capabilities. While it does analyse the problems and barriers faced by new ventures, as well as suggest methods to solve and prevent them, there is little mention of how dynamic capabilities achieve new capabilities. Thus, future research should focus more on how capabilities are developed. Second, any new studies should focus less on the specific characteristics of ventures and instead look at the topic in a more general way that fits the growth models of firms. Third, future research should look at the external factors affecting new ventures, such as capital and product markets, which have a big impact on the development of a company.

Overall, it is important for a company to effectively align IT with business strategy. The perspectives for analysing strategic alignment are: intended alignment, strategic alignment process, and implemented alignment. The dimensions of dynamic capabilities are processes, positions, and paths. A lack of alignment limits the development of IT during business growth. Dynamic capabilities are used to narrow the gap between intended and implemented alignment. Path dependence decreases with sufficient resources, a more long- term view, and dynamic capabilities. 


\section{Application of an integrated circuit in reversing} paralysis

There are many applications of integrated circuit (IC), one being in the field of reversing paralysis. For example, helping paralysed patients regain mobility. But what does reversing paralysis mean? A significant field of medicine, reversing paralysis technology relies on a brain computer interface (BCI) (Fig-1).

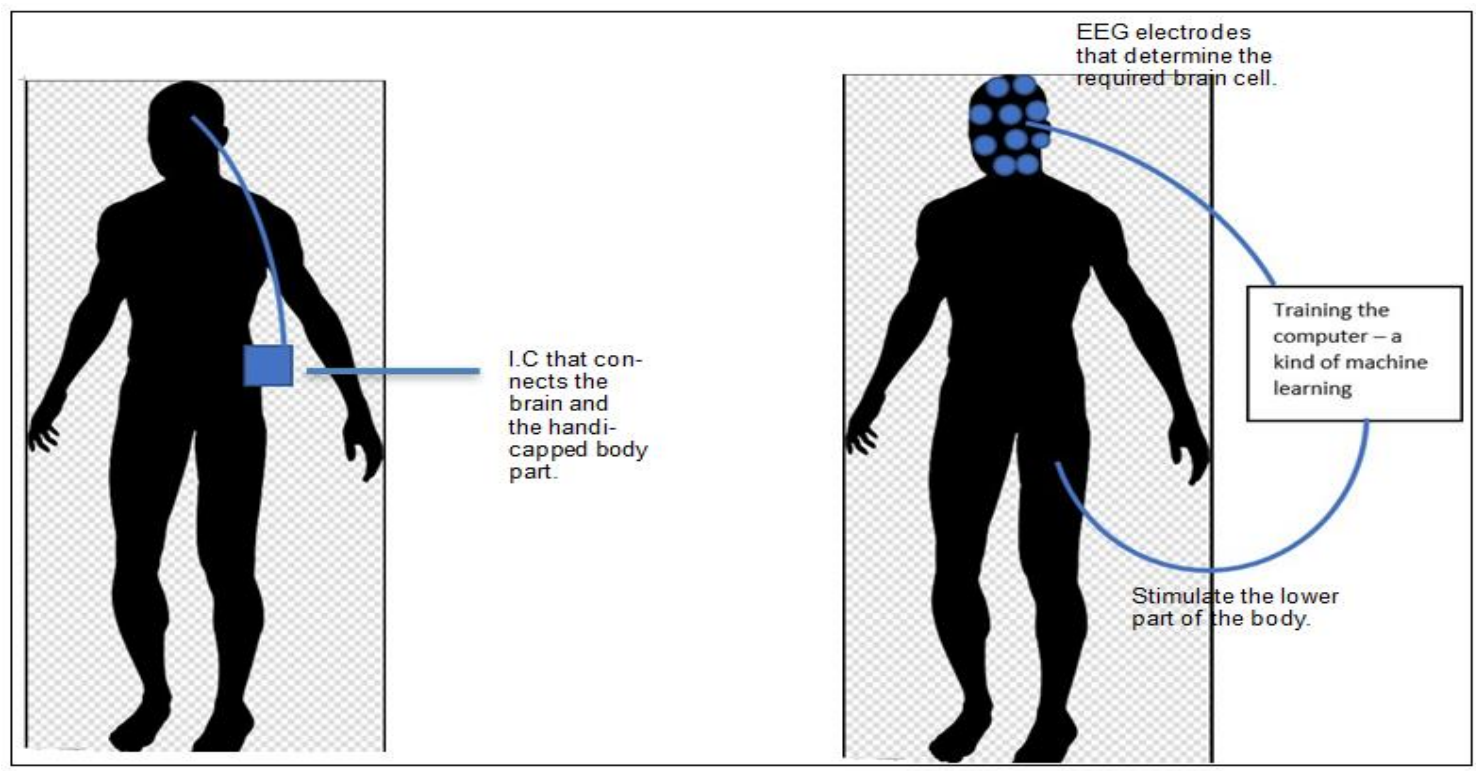

Fig-1: The symmetric usage in reversing paralysis approach

Based on traditional reversing paralysis technology by Hasin [2] and using the electroencephalogram (EEG) for machine learning to stimulate movement in disabled patients [3], this author suggests a symmetric reversing paralysis approach; on that is closely related to the author's earlier net-seizing theory [4].

As mentioned in Chapter 15, the human brain can be considered a type of Bayesian tree that is linked with an IC to the spinal cord. The IC receives signals from the brain and stimulates the relevant part of the

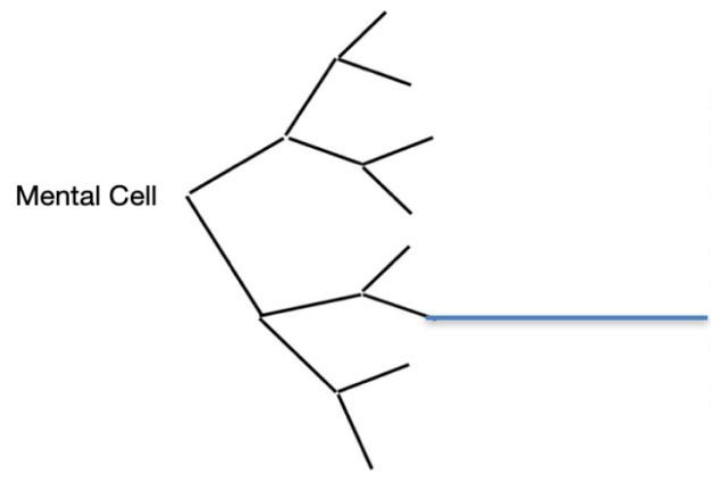

body to move (e.g., curling one's toes). For example, the brain's conscious (mental) cells or neurones first creates the idea about curling your toes. The conscious idea is then carried through the different networked branches of movement cells until the cell specialising in curling your toes — "A move" cell. The A move cell's instruction is decoded and emitted as an electrical signal (from the electronic transmitter) which is then send to the IC located in the spinal cord. When the IC in the spinal cord has received the curling toes signal, the IC will stimulate the lower part of the body to curl your toes.

I.C. that

catches the

brain signal

about the

movement of

the toes and

emits the

signal to

Patient's toes

simulate the

patient's toes

to move.

Fig-2: How a mental cell influences the movement of our body

However, as Altahat [3] explains, EEG signals are highly influenced by the effects of external inputs on the body, such as drinking alcohol. In fact, the alcoholic drink can cause illusion in our brain like the one cannot walk in a straight line. As such, it is important to determine which mental cell — the "A mental" cell corresponds to the initialisation of a mental illusion. To do so, an experiment which applies several EEG electrodes to different areas of the head is recommended. The resulting data can then be used in 
computer learning in order to eliminate the electrical signal errors that may be affected by different mental conditions. There may be a differences in the output EEG results when a patient is measured before and after an alcoholic drink. These EEG electrodes can therefore be considered as the ending point in the reverse branches of a Bayesian tree. This event is because when one is taking alcoholic drink (the action), this action has influenced to the body cells and finally affects the mental cell A that makes decision (due to the illusion that has been influenced to our body before). It is therefore there is a need to search for the corresponding mental task cell: "A mental" during the alignment of our IC implanted system as shown in Figure-3.

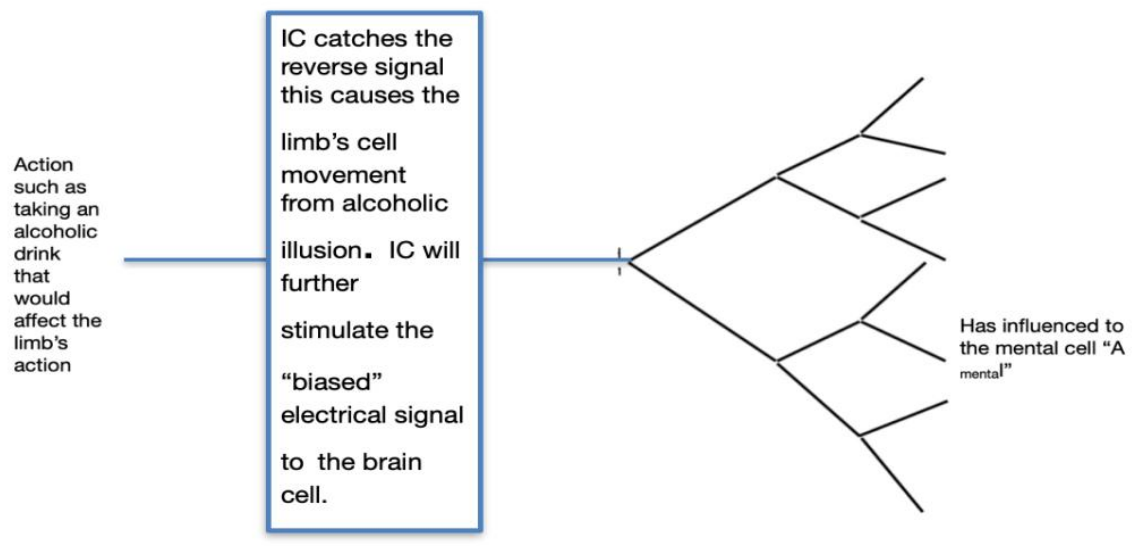

\section{Fig-3: How an action (such as alcoholic drink) causes illusion and influences a brain cell (and finally affects the brain's decision) — the concept of my reverse net-seizing theory}

Figure 2 and Figure 3 show us one of the application to my net-seizing theory (which is a methodology) - a form of philosophy in the field of reversing paralysis technology.

\section{CONCLUSION}

Nowadays ICs have many applications, for example, in electronics (mobile phones) and in the medical field (reversing paralysis). At the same time, it is interest to have on the development of semiconductor industry in Asian countries such as Taiwan, South Korea, Singapore and Hong Kong in 1980s.

This paper provides a brief overview of the development of a Taiwan semiconductor company and the difficulties that it faces. The greatest contribution of the paper, however, is the application of the author's own net-seizing theory to the area of reversing paralysis through a so-called symmetric reversing paralysis approach. Although, it may not be ethical in turning a disabled human into a "semi-human-machine" type of organism, it would be better to help those disabled walking or moving before there is any break-through in our regenerative medicine. It is no doubt that the best way for our body handicapped peoples to move again is through the regeneration in the concerned neurones (cells). However, up to our present time, such kind of the reborn technology has not been ready and need much further development together with experiments. Thus, in the mean time, this author suggests that the best way to aid the disabled people moving again is through my symmetric usage in reversing paralysis philosophy - the implantation of an IC into disabled body for the connection between the brain and the handicapped part.

\section{Remarks}

One may use $3 \mathrm{D}$ printing with stem cells on the surface but (biodegradable polyester as the inner nerve guidance conduit) for the cultivating of regenerated nerve cell to overcome the broken ones. This event can reconnect the paralysed parts like legs and arms that are seriously affected by the accidentally broken nerves. When one goes ahead a step, one can connect the body's limbs with artificial synapse with a "brain-on-a-chip" hardware to the (proposed) artificial brain. The case is just like the famous Hong Kong made robot - Sophia that may be linked with the stem cells on the surface and the biodegradable materials inside (for cultivating the regenerated nerve cells) and the artificial synapse chips on the body to her limbs, then she can have arms and legs with mobility. Hence a semi-bio-robot will be well established.

Beside the application of the proposed netseizing theory in reverse paralysing, one can also apply it into the topic of language translation with the opportunities in finding our origin language. It is well known that one can always translate people's different languages through statistical translation machine. This author proposes that one may also employ the above translation with amendments through the processes like the aforementioned procedures — Bayesian Inference, Bayesian regression and the regression tree. Firstly, one should compute the corresponding Bayesian tree (or the neutral network) for rare words from the context 
independent transformation. Then, one should find out the corresponding linear transformation. Indeed, the meaning of the specific rare word is found according to the domino effects. A sample is the change in part of speech with head node that may lead to the change in the part of speech with all modifiers. Hence, one may predict all of the feasible translated statements that corresponding to the outputted regression tree. Finally, with the help of the business decision theory like our Game theory, one may select the best case of translation such as the one in the result of my decision making thought experiment. Conversely, when one applies the rhetorical unit analysis to a conversation, one may find that different dialogs corresponding to different functions. They are resumed action, prediction, observation, recount and commentary etc [5]. These rhetorical units correspond to different parts of speech and thus give the suitable domino causal effects. If we can find the necessary linear transformation, one may find the required inverse Bayesian tree with the respective conditional probabilities for different meanings to the investigated rare word. The case is like the one shown for the cancer treatment. Thus, my proposed net-seizing theory is the core of the interest in this kind of language translation.

At the same time, one may also employ the language evolution tree with the human historic development [6] (and the genes analysis [7]) to predict the probability of the origin of a particular ethnic group (details are similar to the remarks that shown about the trapping the linear transformation and precision medicine of cancer treatment in Lam March, 2020 [8]. Finally, one can evaluate the feasibility of the only one ancestor in language of our human beings - Homo sapiens as suggested by the Stanford University Professor, Joseph Greenberg [9]. By understanding the origin of our language, it is hope that one may apply the machine translation for the paralysed patients' thought into a spoken language. In other words, less errors will be generated from the translation of patient's nerves. Similar cases can be applied in finding out the origin of a particular virus [10] (e.g., COVIDS-19) through the investigation of its evolution tree [11]. When one goes ahead a step, one may even predict the feasible mutation of such virus by the method of machine learning $[12,13]$. Then one may develop the corresponding future vaccines or drugs for the next generation COVIDS-19 virus.

\section{REFERENCES}

1. Fuller-Love, N., \& Cooper, J. (2000). Deliberate versus emergent strategies: a case study of information technology in the Post Office. International Journal of Information Management, 20(3), 209-223.

2. Hasin, F. (2018). Reversing Paralysis Technology - Remarkable Breakthrough for the Treatment of Incurable Paralysis.

3. Altahat, S. H. Q. (2017). Robust EEG Channel Set for Biometric Application, $\mathrm{PhD}$ thesis, University of Canberra, Australia.

4. Chen, Y., Lam, J. W., Kwok, R. T., Liu, B., \& Tang, B. Z. (2019). Aggregation-induced emission: fundamental understanding and future developments. Materials Horizons, 6(3), 428-433.

5. Ghadessy, M. (1999). Text and Context in Functional Linguistics. Amsterdam; Philadelphia: J. Benjamins. Print. Amsterdam Studies in the Theory and History of Linguistic Science. Ser. IV, Current Issues in Linguistic Theory, 5, 169.

6. https://www.ncbi.nlm.nih.gov/pmc/articles/PMC3 049109/

7. https://www.nature.com/articles/srep22157

8. Lam, W. W., Loke, K. S., Wong, W. Y., \& Ng, D. C. (2020). Facing a disruptive threat: how can a nuclear medicine service be prepared for the coronavirus outbreak 2020?. European Journal of Nuclear Medicine and Molecular Imaging.

9. https://www.angmohdan.com/the-root-of-allhuman-languages/

10. https://www.ncbi.nlm.nih.gov/pmc/articles/PMC3 094976/

11. https://www.ncbi.nlm.nih.gov/pmc/articles/PMC7 $149781 /$

12. https://www.ncbi.nlm.nih.gov/pmc/articles/PMC4 867776/

13. https://www.nature.com/articles/s41746-0190191-0. 